\title{
Sevoflurane downregulates interleukin-6 and interleukin-8 levels in patients after cardiopulmonary bypass surgery: a meta-analysis
}

\author{
Q.B. $Y u^{1}$, H.M. Li ${ }^{1}$, L.L. $\mathrm{Li}^{1}$, S.Y. Wang ${ }^{1}$ and Y.B. $\mathbf{W u}^{1}$ \\ ${ }^{1}$ Department of Cardiosurgery, Fuwai Hospital, \\ Chinese Academy of Medical Science and Peking Union Medical College, Peking, \\ Beijing, China \\ Corresponding author: Y.B. Wu \\ E-mail: wuyongbo1013@163.com \\ Genet. Mol. Res. 14 (4): 19016-19027 (2015) \\ Received August 13, 2015 \\ Accepted October 2, 2015 \\ Published December 29, 2015 \\ DOI http://dx.doi.org/10.4238/2015.December.29.9
}

ABSTRACT. This study aimed to investigate the effect of sevoflurane on serum levels of interleukin (IL)- 6 and IL-8 in patients who underwent cardiopulmonary bypass (CPB). The strength of the association between sevoflurane treatment and serum level of IL- 6 and IL-8 was determined in patients who underwent CPB by summary standard mean differences (SMDs); 95\% confidence interval (Cl) was used. In total, seven casecontrol studies showed decreased IL- 6 and IL-8 levels in sevofluranetreated patients than in controls (IL-6: SMD $=1.56,95 \% \mathrm{Cl}: 0.95-2.17, \mathrm{P}$ $<0.001$; IL-8: SMD $=2.17,95 \% \mathrm{Cl}: 1.40-2.95, \mathrm{P}<0.001$, respectively). Further, IL-6 and IL-8 levels were significantly higher in sevofluranetreated patients than in sevoflurane-pretreated patients (IL-6 post vs pre: $\mathrm{SMD}=2.17,95 \% \mathrm{Cl}: 1.40-2.95, \mathrm{P}<0.001 ; \mathrm{IL}-8$ post vs pre: $\mathrm{SMD}=4.01$, $95 \% \mathrm{Cl}: 2.80-5.21, \mathrm{P}<0.001$, respectively). CPB-stratified analysis showed significant decrease in IL-6 and IL-8 levels in sevoflurane-treated patients than in controls, irrespective of the time after CPB surgery $(P<0.05)$. 
Moreover, sevoflurane-pretreated patients under the $<12-\mathrm{h}$ subgroup showed decreased IL-6 levels $(P=0.698)$, while all other subgroups showed decreased IL-8 levels $(P<0.05)$. Further, subgroup analysis by different dose of sevoflurane showed decreased IL-6 and IL-8 levels in subgroups administered with a dose of $<2$ and $\geq 2 \%$ sevoflurane under the case vs control and pre- vs post-treatment of sevoflurane models. Serum IL-6 and IL-8 levels were significantly lower in sevoflurane-treated patients who underwent $\mathrm{CPB}$, suggesting sevoflurane pretreatment to be more beneficial than post-treatment.

Key words: Interleukin-6; Interleukin-8; Sevoflurane; Meta-analysis; Cardiopulmonary bypass

\section{INTRODUCTION}

Sevoflurane is a new anesthetic agent that is used for inducing and maintaining general anesthesia (Ogurlu et al., 2014). Although anesthetics are important in alleviating surgical pain in patients, they also contribute to postoperative conditions such as agitation, excitement, nausea, and vomiting, which are also seen in sevoflurane inhalation (Yin et al., 2014). Sevoflurane has several beneficial effects that are actively pursued for their clinical application. For example, sevoflurane treatment in breast cancer patients may increase IL-10 levels to inhibit the production of various pro-inflammatory cytokines such as interleukin (IL)-1/-6/-8, tumor necrosis factor (TNF)- $\alpha$, and matrix metalloproteinases (MMPs), suggesting the clinical application of sevoflurane in immunomodulation (Deegan et al., 2010). Cardiopulmonary bypass (CPB) is associated with myocardial ischemia-reperfusion injury that results in high production of proinflammatory cytokines such as IL-6 and IL-8. Here we analyze the sevoflurane-mediated effects on proinflammatory mediators in CBP settings, as it is suggested that sevoflurane might suppress inflammation (Cho et al., 2009).

IL-6 is a cytokine that is produced by T cells and macrophages exhibiting either pro- or anti-inflammatory properties (Zhang et al., 2014). Pro-inflammatory role of IL-6 mediates immune responses after infection and trauma, possibly resulting in massive tissue injuries (Lenski and Scherer, 2014). IL-8 is a chemokine mainly secreted by epithelial, endothelial, and smooth muscle cells and macrophages, and plays an important role in the innate immune system (Zarogoulidis et al., 2014). IL-8 is also known as neutrophil chemotactic factor that induces chemotaxis of target cells by causing neutrophils and granulocytes to migrate toward the site of infection (Sunaga et al., 2014). IL-6 and IL-8 levels in patients during coronary artery bypass grafting (CABG) sharply increase from normal at the time of surgery, to its highest levels by the end of surgery. Elevated levels of IL- 6 and IL- 8 may have fatal influence on pulmonary function resulting from endocrine stress response and inflammatory activation (Winterhalter et al., 2008). Hence, sevoflurane involvement modulates IL-6 and IL-8 expression, which may have a potential role in CPB patients. Sevoflurane is administered as a premedication during CPB and can inhibit the expression and release of tumor necrosis factor (TNF), thus contributing to the suppression of IL-6 and IL-8 production, regulating the activation of inflammation, and eventually may result in the protection of myocardial function (Schmid et al., 2012). Hence, sevoflurane treatment is closely associated with the serum levels of IL-6 and IL- 8 in patients with CPB (Nader et al., 2006; Wang et al., 2013); however, results from previous studies are inconsistent with this hypothesis (Zhang et al., 2007; Ma et al., 2009). Therefore, we conducted a comprehensive meta-analysis to investigate sevoflurane effect on IL-6 and IL-8 levels in patients with CPB. 


\section{METHODS}

\section{Data sources and keywords}

This meta-analysis was conducted and reported according to the PRISMA guidelines (as shown in Annex I). Studies published before 30 June 2014 were selected based on the correlation between sevoflurane treatment and serum level of IL-6 and IL-8 in patients who underwent CABG with CPB. Computerized literature databases such as Embase, Web of Science, Google Scholar, PubMed, China BioMedicine (CBM) and China National Knowledge Infrastructure (CNKI) were searched by combining medical subject headings (Mesh) and keywords for sevoflurane ("sevoflurane" or "sevoflurane" or "sevorane" or "Ultane") and ILs ("Interleukins" or "IL"). No restriction was set for the published languages or dataset. A manual search using cross-references also retrieved additional articles. When data were not clear in the original article, authors were contacted for clarifications.

\section{Study selection}

Studies selected in this meta-analysis conformed to the following inclusion criteria: 1) case-control trials within a human population to explore the association of sevoflurane treatment with the serum levels of IL-6 and IL-8 in patients underwent CPB were incorporated; 2) only those participants who underwent CPB were included; 3 ) study subjects were divided into two groups: control, who received fentanyl and propofol instead of sevoflurane, and cases, who received fentanyl and sevoflurane; 4) data from the included articles must be original; 5) this meta-analysis evaluates the alternations in IL-6 and IL-8 levels, so sufficient information must be provided regarding the serum levels of IL-6 and IL-8. The exclusion criteria were: 1) those that did not satisfy the inclusion criteria; 2) abstracts, case report, letters, proceedings, or meta-analysis; 3) duplicated papers or studies with overlapping data.

\section{Data extraction}

For the purpose of reducing bias and enhancing confidence, two investigators (HM L and LL L) independently selected and evaluated the studies based on the selection criteria. Discussion and reexamination was used as a format to resolve disagreements (SY W). In addition to the measurement of outcomes, the following relevant data were extracted: 1) surname of first author and time of publication; 2) country and ethnicity of publication; 3) number of samples, age, and sex of subjects; 4) detection method for serum IL-6 and IL-8 levels; 5) follow-up time; 6) preoperative and postoperative serum levels of IL-6 and IL-8. Ratings were completed by two independent reviewers (HM L and LL L), with a third independent reviewer (SY W) resolving disagreements.

\section{Quality Assessment}

Two authors independently used a set of predefined criteria based on the NewcastleOttawa scale (NOS) criteria to decide whether the study in question is of high quality (Stang, 2010). The following three grades were set: 1) subject selection: $0-4$; 2) comparability of subject: $0-2 ; 3)$ clinical outcome: 0-3. Total NOS scores ranged from 0-9 (lowest to highest), and there were two levels: low quality (0-6), high quality (7-9), respectively. Each item was assessed and finally judged as low or high. 


\section{Statistical analysis}

Meta-analysis of effect sizes was performed using the STATA software, version 12.0 (Stata Corp, College Station, TX, USA). All results with $\mathrm{P}<0.05$ were regarded statistically significant. The strength of the association between sevoflurane treatment and serum level of IL-6 and IL-8 was determined in patients who underwent CPB by summary standard mean differences (SMDs); $95 \%$ confidence interval $(\mathrm{Cl})$ was used to gauge the precision of the summary SMD. The significance of the summary SMD was determined using Z-test. Quantitative analysis was performed with $95 \% \mathrm{Cl}$ using a random-effects model (DerSimonian and Laird method) or a fixed-effects model (Mantel-Haenszel method) to minimize the variance of the summary SMDs. Random-effects model was applied when significant heterogeneity existed among studies, or we chose the fixedeffects model. Cochran's Q-statistic was used to evaluate the heterogeneity across the studies. Because the Cochran's Q-statistic has low statistical power, $I^{2}$ test $(0 \%$, no heterogeneity; $100 \%$, maximal heterogeneity) was also used (Peters et al., 2006; Jackson et al., 2012). For evaluating the extent of heterogeneity, stratified subgroup analyses by time point after CPB was conducted to explore potential effect modification. Funnel plot was constructed to investigate whether publication bias affects the validity of the overall estimation (Zintzaras and loannidis, 2005). A symmetric figure may indicate no possible existence of publication bias. Egger's linear regression test measured the symmetry of the funnel plot (Egger et al., 1997) using the $t$-test, and $P<0.05$ showed statistical significance of publication bias.

\section{RESULTS}

\section{Included studies}

A total of seven papers, published between 2004 and 2013, out of 288 relevant articles identified by computerized databases and manual searching methods, met our inclusion criteria by providing information on the association of sevoflurane treatment with the serum level of IL- 6 and IL-8 in patients who underwent CPB (Nader et al., 2004, 2006; Zhang et al., 2007; Ma et al., 2009; Gao et al., 2012; Mao et al., 2012; Wang et al., 2013). Methodological quality of the extracted studies is presented in Table 1. A total of five studies were performed in Asians and two in Caucasians involving 192 subjects, in China and USA. The range of dose distributions of sevoflurane was 1 3\%. Enzyme linked immunosorbent assay (ELISA) was used to detect serum levels of IL-6 and IL-8 pre- and post-treatment of sevoflurane. The steps for screening and the study selection procedure are given in Figure 1.

\section{Association of sevoflurane with serum IL-6 and IL-8 levels in CPB patients}

The pooled SMDs for serum IL-6 and IL-8 levels revealed that their serum levels were significantly lower in the case groups as compared to normal controls (IL-6: SMD = 1.56, 95\% Cl: 0.95-2.17, $\mathrm{P}<0.001$; IL-8: SMD = 2.17, 95\% Cl: 1.40-2.95, $\mathrm{P}<0.001$; respectively), as given in Figure 2. Patients with $\mathrm{CPB}$ who received post-treatment of sevoflurane showed higher serum IL-6 and IL-8 levels than those who received pretreatment of sevoflurane (IL-6: SMD $=1.63,95 \% \mathrm{Cl}: 0.30-2.96, \mathrm{P}=0.016 ; \mathrm{IL}-8: \mathrm{SMD}=4.01,95 \% \mathrm{Cl}: 2.80-5.21, \mathrm{P}<0.001$; respectively). 


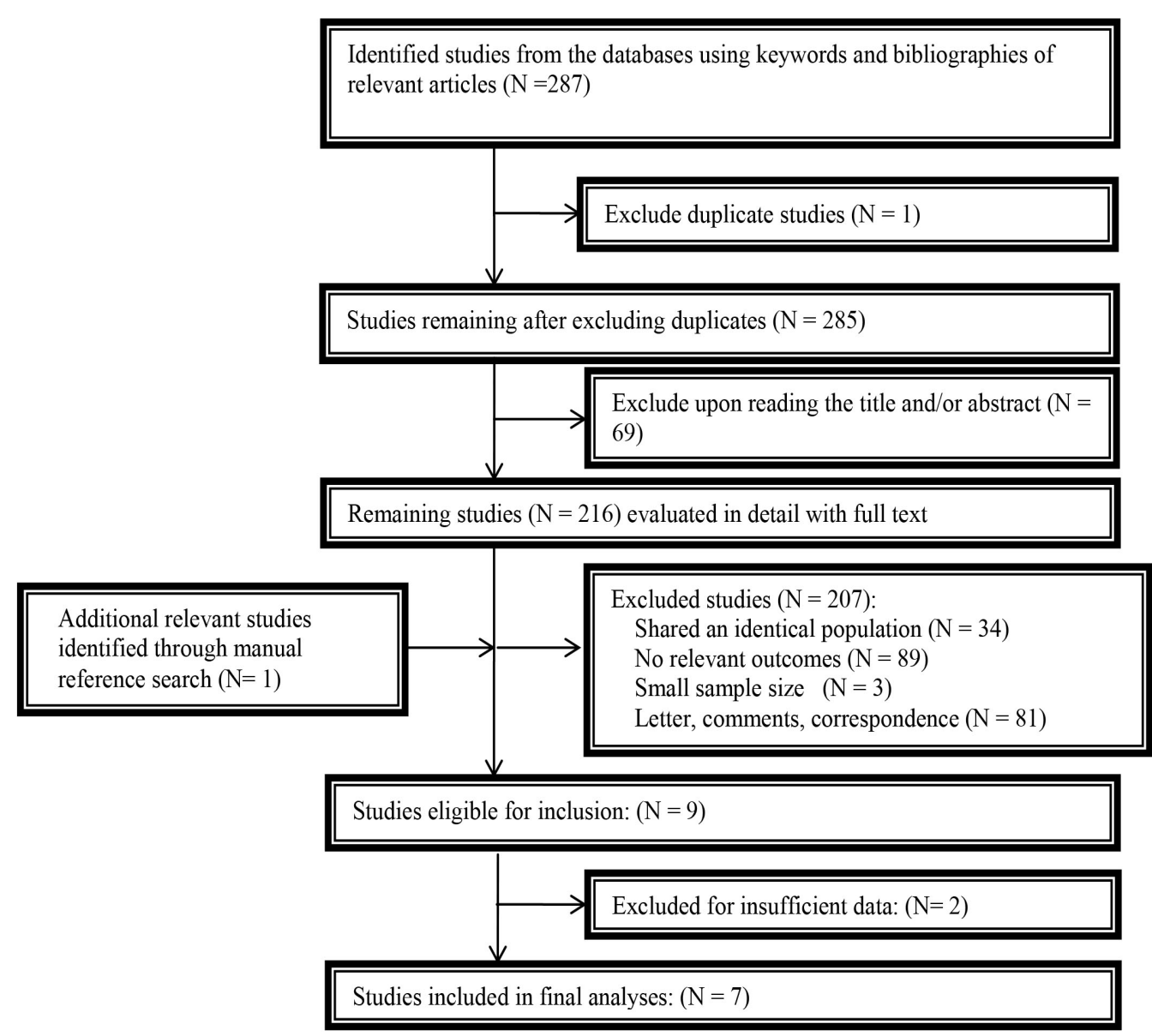

Figure 1. Flow chart showing study selection procedure. This meta-analysis involved seven studies.

\begin{tabular}{|c|c|c|c|c|c|c|c|c|c|}
\hline \multirow[b]{2}{*}{ Study } & \multicolumn{4}{|c|}{ Selection (score) } & \multirow{2}{*}{$\begin{array}{l}\text { Comparability (score) } \\
\text { - Control for } \\
\text { important } \\
\text { or factor or }\end{array}$} & \multicolumn{4}{|c|}{ Exposure (score) } \\
\hline & $\begin{array}{c}\text { Adequate } \\
\text { definition } \\
\text { of patient case }\end{array}$ & $\begin{array}{l}\text { Representativeness } \\
\text { of patient cases } \\
\text { a }\end{array}$ & $\begin{array}{l}\text { Selection } \\
\text { of controls }\end{array}$ & $\begin{array}{c}\text { Definition } \\
\text { of controls } \\
\text { additional factor }\end{array}$ & & $\begin{array}{l}\text { Ascertainment of } \\
\text { of exposure } \\
\text { (blinding) }\end{array}$ & $\begin{array}{l}\text { Same method } \\
\text { ascertainment } \\
\text { for participants }\end{array}$ & $\begin{array}{c}\text { Nonresponse } \\
\text { rate }^{\star}\end{array}$ & $\begin{array}{l}\text { Total } \\
\text { score** }\end{array}$ \\
\hline ang YW & 1 & 1 & 1 & 1 & 1 & 0 & 1 & 0 & 6 \\
\hline Mao QQ & 1 & 0 & 1 & 1 & 0 & 1 & 1 & 0 & 5 \\
\hline Gao CJ & 1 & 1 & 1 & 1 & 0 & 1 & 1 & 1 & 7 \\
\hline Ma J & 1 & 0 & 1 & 1 & 1 & 0 & 1 & 1 & 6 \\
\hline Zhang XL & 1 & 1 & 1 & 1 & 1 & 0 & 1 & 0 & 6 \\
\hline Nader ND & 1 & 1 & 1 & 1 & 0 & 1 & 1 & 1 & 7 \\
\hline Nader ND & 1 & 1 & 1 & 1 & 1 & 0 & 1 & 1 & 7 \\
\hline
\end{tabular}

*When there was no significant difference in the response rate between both groups by using $X^{2}$ test $(P>0.05)$, one point was awarded. ${ }^{* *}$ Total score could range from 0 to 9 points. 


\section{IL-6 serum level (Case vs Control)}

\begin{tabular}{|c|c|c|c|}
\hline ncluded study & & SMD $(95 \% \mathrm{Cl}) \quad \mathrm{W}$ & Neight $\%$ \\
\hline Wang YW (2013) & 1 & $1.70(0.97,2.43)$ & 5.84 \\
\hline Mao QQ (2012) & ; & $19.29(14.91,23.67)$ & i7) 1.49 \\
\hline Gao CJ-a (2012) & H & $2.26(1.11,3.41)$ & 5.20 \\
\hline Gao CJ-b (2012) & 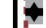 & $2.73(1.48,3.98)$ & 5.03 \\
\hline Gao CJ-c (2012) & s & $3.36(1.96,4.77)$ & 4.76 \\
\hline Gao CJ-d (2012) & A & $1.00(0.07,1.94)$ & 5.54 \\
\hline Ma J-a (2009) & & $0.14(-0.74,1.01)$ & 5.63 \\
\hline Ma J-b (2009) & & $0.34(-0.54,1.22)$ & 5.62 \\
\hline Ma J-c (2009) & 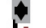 & $0.86(-0.06,1.78)$ & 5.57 \\
\hline Ma J-d (2009) & 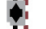 & $0.14(-0.74,1.02)$ & 5.63 \\
\hline Ma J-e (2009) & & $0.67(-0.23,1.58)$ & 5.59 \\
\hline Ma J-f(2009) & 4 & $1.10(0.16,2.05)$ & 5.52 \\
\hline Zhang XL-a (2007) & 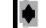 & $1.94(1.06,2.82)$ & 5.63 \\
\hline Zhang XL-b (2007) & 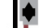 & $1.97(1.09,2.85)$ & 5.62 \\
\hline Zhang XL-c (2007) & c) & $0.98(0.22,1.74)$ & 5.80 \\
\hline Nader ND-a (2004) & $\checkmark$ & $1.12(0.19,2.04)$ & 5.55 \\
\hline Nader ND-b (2004) & & $0.11(-0.75,0.97)$ & 5.66 \\
\hline Nader ND-c (2004) & 4 & $3.76(2.29,5.23)$ & 4.65 \\
\hline Nader ND-d (2004) & 1 & $0.00(-0.86,0.86)$ & 5.66 \\
\hline Heterogeneity test $\left(l^{2}=86.5 \%, p=0.000\right)$ & 4 & $1.56(0.95,2.17)$ & 100.00 \\
\hline$Z$ test $(Z=5.00, P<0.001)$ & 7 & & \\
\hline Random effects analysis & & & \\
\hline-23.7 & 0 & 23.7 & \\
\hline
\end{tabular}

\section{IL-8 serum level (Case vs Control)}

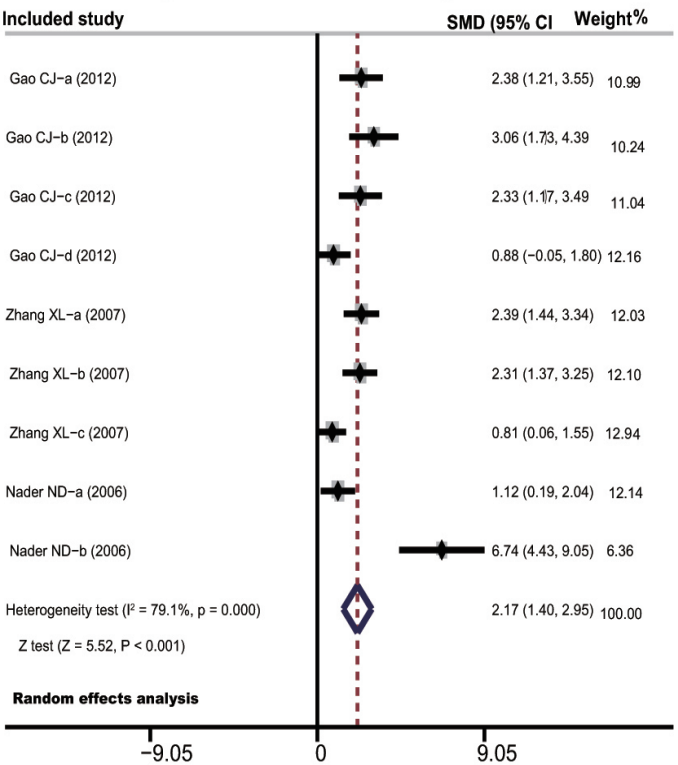

\section{IL-6 serum level (Post vs Pre)}

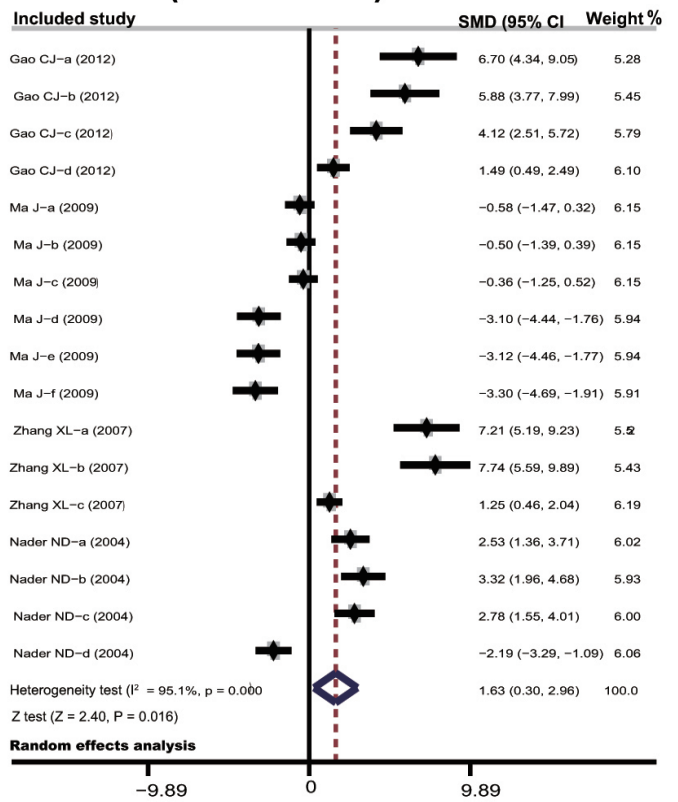

\section{IL-8 serum level} (Post vs Pre)

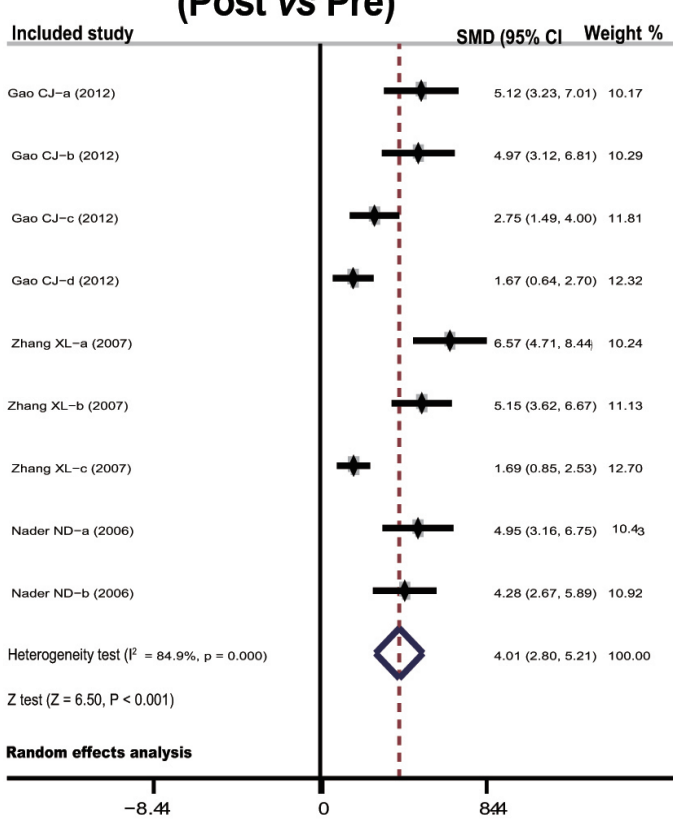

Figure 2. Forest plots showing the difference of serum IL-6 and IL-8 levels between healthy subjects and sevofluranetreated patients after cardiopulmonary bypass surgery via pre- and post-treatment of sevoflurane. 


\section{Subgroup analysis}

Subgroup analysis based on time point after CPB showed that serum IL-6 levels in sevoflurane-treated patients decreased significantly compared to that of control group within both the $<12 \mathrm{~h}$ after CPB (SMD = 1.86, 95\% Cl: 0.99-2.72, $\mathrm{P}<0.001)$ and $>12 \mathrm{~h}$ after CPB groups (SMD $=1.09,95 \% \mathrm{Cl}: 0.42-1.76, \mathrm{P}=0.001)$. Similar results were also found in the comparison of serum IL-8 levels between the cases and controls (all $\mathrm{P}<0.05$ ) (Figure 3). However, serum IL-6 level was found to increase only in the $<12 \mathrm{~h}$ subgroup post-treated with sevoflurane $(\mathrm{SMD}=2.80$, $95 \% \mathrm{Cl}: 1.07-4.53, \mathrm{P}=0.001)$, but not in the subgroup of $>12 \mathrm{~h}$ after $\mathrm{CPB}(\mathrm{SMD}=-0.44,95 \% \mathrm{Cl}$ : -2.66-1.78, $P=0.698$ ). In addition, positive correlation was seen between the serum IL-8 levels of sevoflurane-treated $<12 \mathrm{~h}$ and $>12 \mathrm{~h}$ subgroups (all $\mathrm{P}<0.05$ ). Subgroup analysis with different dose of sevoflurane showed decreased IL-6 and IL-8 levels in sevoflurane-treated patients than in control group with $<2$ and $\geq 2 \%$ doses (all $P<0.05$ ). Moreover, pre- and post-treatment levels of IL-6 and IL-8 decreased in both subgroups of different sevoflurane dose (all P < 0.05) (Figure 4).
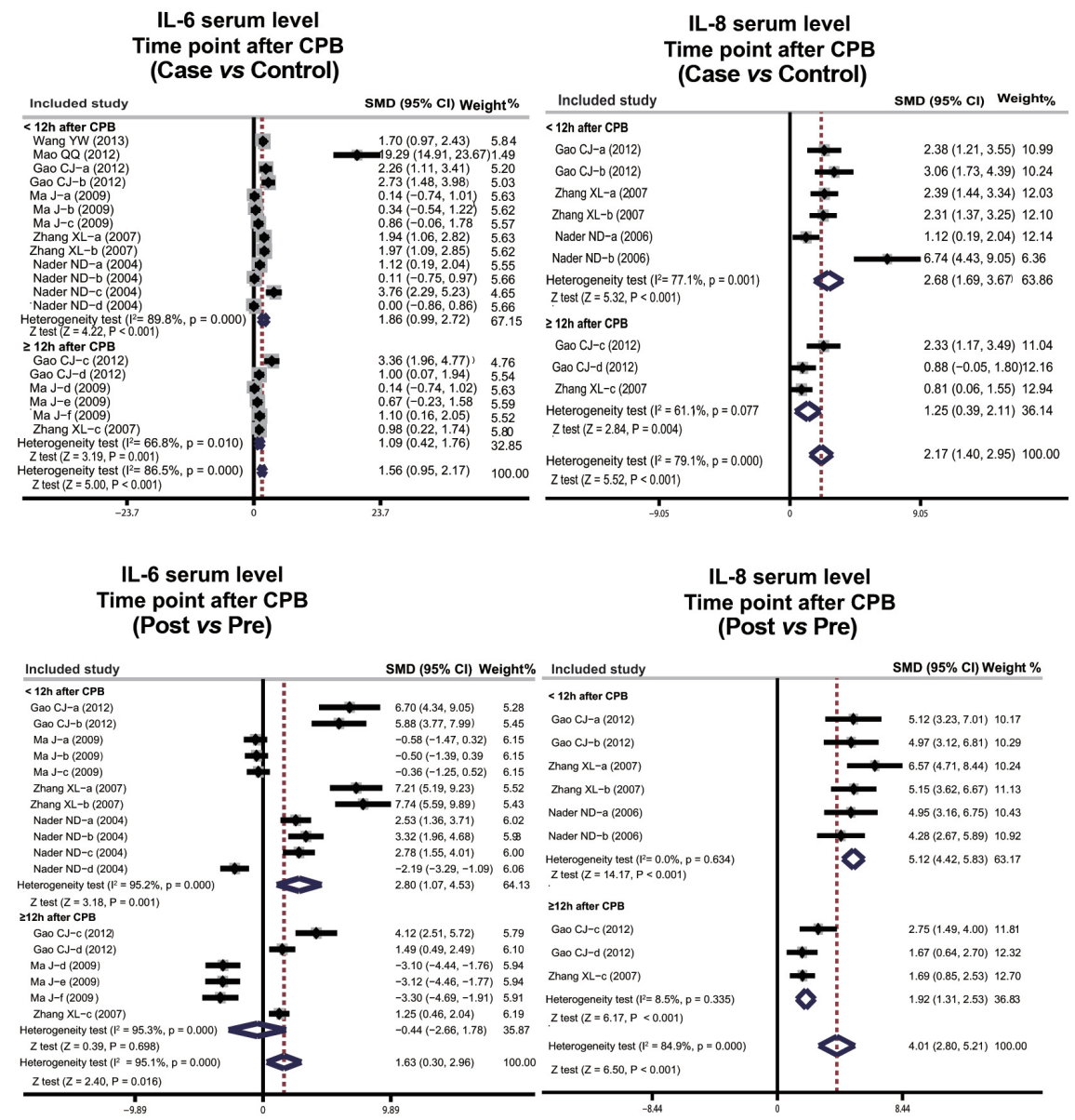

Figure 3. Subgroup analyses by time point on the difference of serum IL-6 and IL-8 levels between healthy subjects and sevoflurane-treated patients after cardiopulmonary bypass surgery via pre- and post-treatment of sevoflurane. 


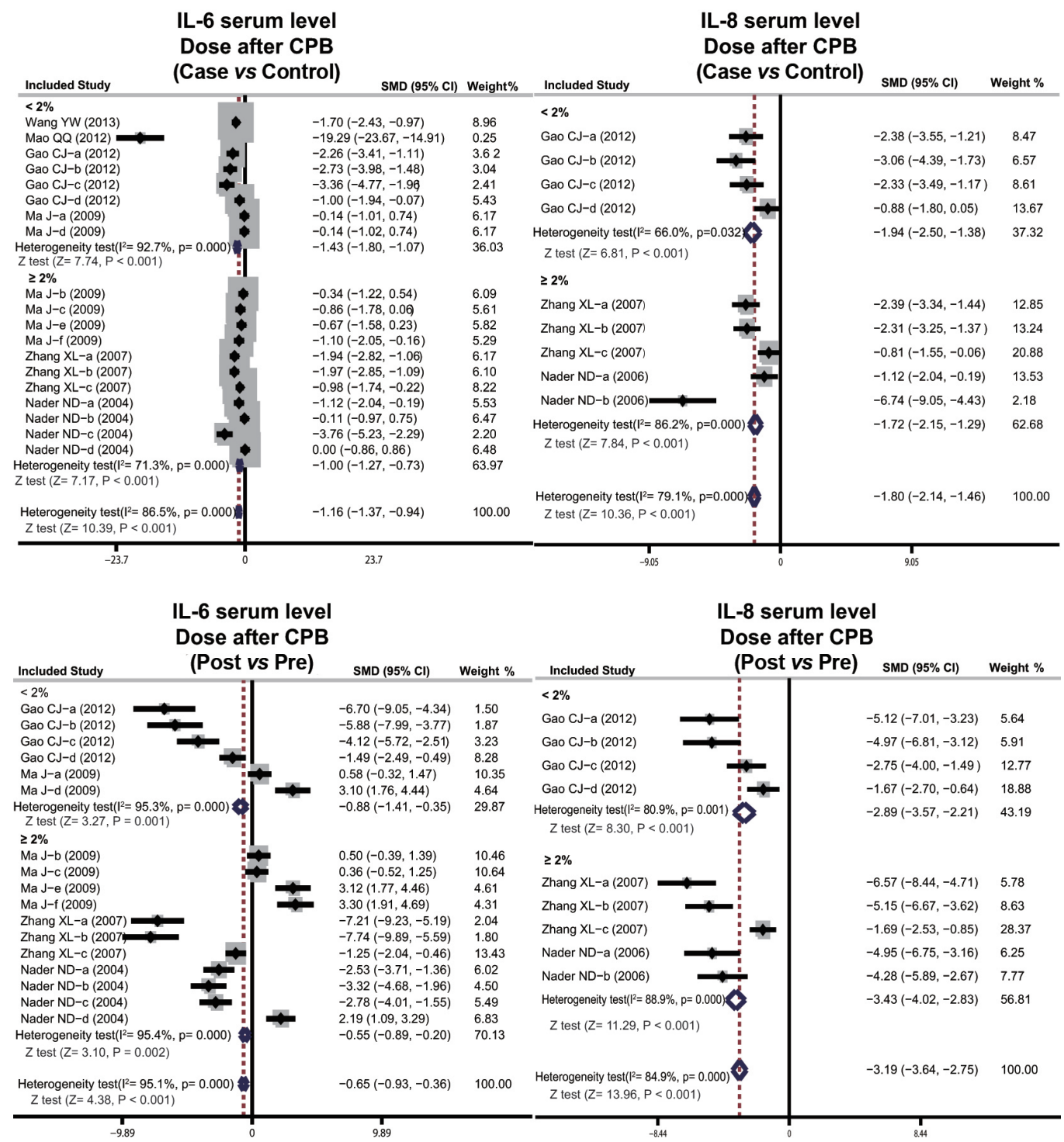

Figure 4. Subgroup analyses by different dose of sevoflurane on the difference of serum IL-6 and IL-8 levels between healthy subjects and sevoflurane-treated patients after cardiopulmonary bypass surgery via pre- and post-treatment of sevoflurane.

\section{Publication bias}

Graphical funnel plots for serum IL-6 and IL-8 levels presented to be asymmetrical. Egger's test suggested a possible existence of publication bias in both the case vs control models (IL-6: $\mathrm{t}=5.82, \mathrm{P}<0.001$; IL-8: $\mathrm{t}=5.34, \mathrm{P}=0.001$; respectively) and pretreatment $v s$ post-treatment models (IL-6: $t=2.33, P=0.034$; IL-8: $t=8.10, P<0.001$; respectively) (Figure 5). 
IL-6 serum level (Case vs Control)

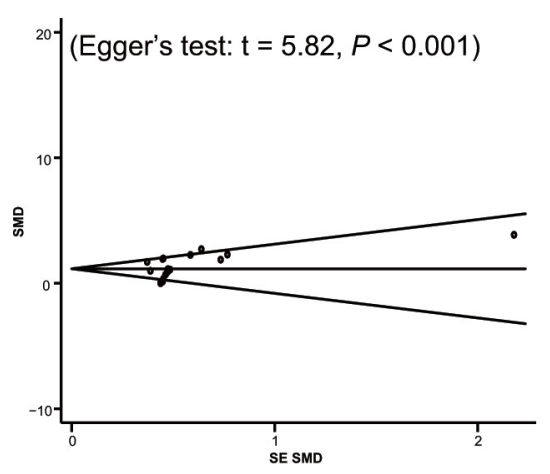

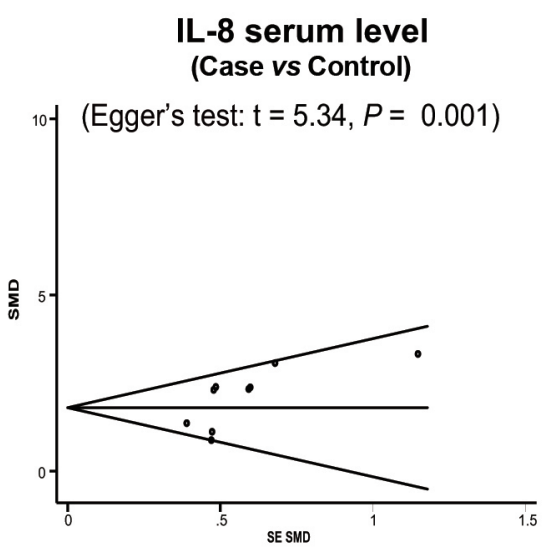

IL-8 serum level Case vs Control) 
play leading roles in increasing the pulmonary endothelial permeability; hence, damage to lung tissue structure is a critical event for initiating an pulmonary edema, atelectasis, or hypoxemia postoperatively (Grommes and Soehnlein, 2011; Muller-Redetzky et al., 2014). Sevoflurane could successfully decrease the production of inflammatory mediators, which is largely attributed to the mechanism of sevoflurane in blocking the activation of the complement system and downregulating the generation of inflammatory mediators, and thereby help to reduce inflammatory response caused by CPB (Schmid et al., 2012; Watanabe et al., 2013; Aguado et al., 2014). Hence, control of inflammatory cytokine levels may help to reduce myocardial and pulmonary injury (Seki et al., 2010; Lee and Yang, 2012).

Further, both pre- and post-treatment of sevoflurane have protective myocardial effects (Anzai et al., 2013).There are significant differences between pretreatment and post-treatment of sevoflurane because of its in vivo mechanisms. With the opening of the ATP-sensitive potassium channel, pretreatment of sevoflurane may contribute to an increased mitochondrial matrix volume and maintenance of the integrity of mitochondria, and eventually exerting its effects on myocardial protection (Bouwman et al., 2007; Onishi et al., 2012). Myocardial ischemia reperfusion injury contributes to the opening of the mitochondrial permeability transition pore (mPTP). This causes damage to the inner mitochondrial membrane, the barrier that separates the intramitochondrial solutes from the extra-mitochondrial environment, which leads to the release of cytochrome $C$ that is related to apoptosis (Kristen et al., 2013; Ong et al., 2015). In addition, post-treatment of sevoflurane may suppress myocardial injury by inhibiting mPTP opening (Li et al., 2013). Generally, sevoflurane pretreatment occurs before ischemia to improve ischemic tolerance; while post-treatment with sevoflurane is focused on the intraoperative or postoperative lung injury during CPB to reduce ischemic injury in the acute phase (Orriach et al., 2013; Zhou et al., 2013). By the suppression of advanced glycation end products synthesis and activation, sevoflurane post-treatment was suggested to eventually contribute to the downregulation of inflammatory mediators (Dong et al., 2014). In this meta-analysis, serum IL-6 and IL-8 levels in patients who received post-treatment of sevoflurane were much higher than in those who received pretreatment of sevoflurane, indicating that pretreatment of sevoflurane might be beneficial to patients after CPB through suppressing the release or expression of inflammatory cytokines and thereby reducing the degree of inflammation after CPB.

Further subgroup analysis by time point after CPB implicated that the serum IL-6 and IL-8 levels in sevoflurane-pretreated patients decreased more significantly than those in the control group within the $<12 \mathrm{~h}$ after CPB and $>12 \mathrm{~h}$ after CPB groups. However, post-treatment of sevoflurane the serum IL- 6 levels only decreased in the $<12$ h after CPB group, but not in the subgroup of $>12$ $\mathrm{h}$ after CPB. This result suggests that sevoflurane may have short-term effect in improving cardiac function after weaning from CPB, but showed no long-term effect. This hypothesis, however, needs further investigation in more patients. Additionally, subgroup analysis by different doses of sevoflurane showed that both IL-6 and IL-8 levels decreased in sevoflurane-treated patients than that of control group in the dose $<2$ and $\geq 2 \%$ subgroups, and there were no difference of the serum levels of IL- 6 and IL-8 under the model of pre- and post-treatment of sevoflurane. These results suggest that different doses of sevoflurane may have no significant influence in the efficacy of sevoflurane.

We understand the limitations of the current meta-analysis. First, there were only seven articles enrolled and most were conducted in Asians. Moreover, the number of patients involved in the study was also too small to predict valid conclusion in the meta-analysis, which might cause selection bias. Furthermore, cytokines might be readily released during or after heart surgery, suggesting the need of a much larger and homogeneous population for valid conclusions. Second, subgroup analysis (time point after CPB) was relatively small and further excluded potential 
heterogeneity sources that affect our results. Other factors (e.g., ethnicity, country, sample size, or dosage) should also be further explored. Third, there was no further dose effect of sevoflurane investigation on the levels of IL-6 and IL-8 in patients with CPB, which clearly restricted the wider applicability of our results. Finally, although we wanted to study the effects of sevoflurane on ischemia-reperfusion injury, there were only two cytokines (IL-6/IL-8) studied, which was not enough to answer the question we posed. Several other parameters should be included in the meta-analysis to provide a more comprehensive assessment.

In conclusion, serum IL-6 and IL-8 levels were significantly lower after sevoflurane treatment in patients who underwent $\mathrm{CPB}$, and pretreatment with sevoflurane might be more beneficial than post-treatment of sevoflurane. Future investigation is needed to interpret the association between sevoflurane treatment and the enrollment of more parameters during intra-cardiac surgery with CPB.

\section{CONFLICTS OF INTEREST}

The authors declare no conflict of interest.

\section{ACKNOWLEDGMENTS}

We would like to acknowledge the reviewers for their helpful comments on this paper.

\section{REFERENCES}

Aguado D, Abreu M, Benito J, Garcia-Fernandez J, et al. (2014). Amitriptyline, minocycline and maropitant reduce the sevoflurane minimum alveolar concentration and potentiate remifentanil but do not prevent acute opioid tolerance and hyperalgesia in the rat: a prospective, randomised laboratory study. Eur. J. Anaesthesiol. 32: 248-254.

Anzai M, lijima N, Higo S, Takumi K, et al. (2013). Direct and specific effect of sevoflurane anesthesia on rat Per2 expression in the suprachiasmatic nucleus. PLoS One 8: e59454.

Bouwman RA, Musters RJ, van Beek-Harmsen BJ, de Lange JJ, et al. (2007). Sevoflurane-induced cardioprotection depends on PKC-alpha activation via production of reactive oxygen species. Br. J. Anaesth. 99: 639-645.

Cho EJ, Yoon JH, Hong SJ, Lee SH, et al. (2009). The effects of sevoflurane on systemic and pulmonary inflammatory responses after cardiopulmonary bypass. J. Cardiothorac. Vasc. Anesth. 23: 639-645.

Deegan CA, Murray D, Doran P, Moriarty DC, et al. (2010). Anesthetic technique and the cytokine and matrix metalloproteinase response to primary breast cancer surgery. Reg. Anesth. Pain. Med. 35: 490-495.

Dong P, Zhao J, Zhang Y, Dong J, et al. (2014). Aging causes exacerbated ischemic brain injury and failure of sevoflurane post-conditioning: role of B-cell lymphoma-2. Neuroscience 275: 2-11.

Egger M, Davey Smith G, Schneider M and Minder C (1997). Bias in meta-analysis detected by a simple, graphical test. BMJ 315: 629-634.

Gao CJ, Li B, Wang HX, Lv XM, et al. (2012). Effects of sevoflurane pretreatment on inflammatory response in patients undergoing cardiac valve replacement with cardiopulmonary bypass. Chin. J. Anesthesiol. 2: 1081-1084.

Grommes J and Soehnlein O (2011). Contribution of neutrophils to acute lung injury. Mol. Med. 17: 293-307.

Jackson D, White IR and Riley RD (2012). Quantifying the impact of between-study heterogeneity in multivariate metaanalyses. Stat. Med. 31: 3805-3820.

Kortekaas KA, van der Baan A, Aarts LP, Palmen M, et al. (2014). Cardiospecific sevoflurane treatment quenches inflammation but does not attenuate myocardial cell damage markers: a proof-of-concept study in patients undergoing mitral valve repair. Br. J. Anaesth. 112: 1005-1014.

Kristen AV, Ackermann K, Buss S, Lehmann L, et al. (2013). Inhibition of apoptosis by the intrinsic but not the extrinsic apoptotic pathway in myocardial ischemia-reperfusion. Cardiovasc. Pathol. 22: 280-286.

Lee IT and Yang CM (2012). Role of NADPH oxidase/ROS in pro-inflammatory mediators-induced airway and pulmonary diseases. Biochem. Pharmacol. 84: 581-590.

Lenski M and Scherer MA (2014). The significance of interleukin-6 and lactate in the synovial fluid for diagnosing native septic arthritis. Acta. Orthop. Belg. 80: 18-25. 
Li H, Zhou C, Chen D, Fang N, et al. (2013). Failure to protect against myocardial ischemia-reperfusion injury with sevoflurane postconditioning in old rats in vivo. Acta. Anaesthesiol. Scand. 57: 1024-1031.

Ma J, Fang C, Wang RM, Kang F, et al. (2009). Effect of sevoflurane preconditioning on protecting lung injury in patients undergoing cardiac surgery with cardiopulmonary bypass. Shandong Med. J. 91-93.

Mao QQ, Zou J and Zhang ZJ (2012). Effect of sevoflurane preconditioning on inflammatory response in 40 patients undergoing cardiac surgery with cardiopulmonary bypass. Chongqing Med. 41: 1975-1976.

Muller-Redetzky HC, Suttorp N and Witzenrath M (2014). Dynamics of pulmonary endothelial barrier function in acute inflammation: mechanisms and therapeutic perspectives. Cell Tissue Res. 355: 657-673.

Nader ND, Li CM, Khadra WZ, Reedy R, et al. (2004). Anesthetic myocardial protection with sevoflurane. J. Cardiothorac. Vasc. Anesth. 18: 269-274.

Nader ND, Karamanoukian HL, Reedy RL, Salehpour F, et al. (2006). Inclusion of sevoflurane in cardioplegia reduces neutrophil activity during cardiopulmonary bypass. J. Cardiothorac. Vasc. Anesth. 20: 57-62.

Ogurlu M, Sari S, Kucuk M, Bakis M, et al. (2014). Comparison of the effect of propofol and sevoflurane anaesthesia on acute and chronic postoperative pain after hysterectomy. Anaesth. Intensive Care 42: 365-370.

Ong SB, Samangouei P, Kalkhoran SB and Hausenloy DJ (2015). The mitochondrial permeability transition pore and its role in myocardial ischemia reperfusion injury. J. Mol. Cell. Cardiol. 78: 23-34.

Onishi A, Miyamae M, Kaneda K, Kotani J, et al. (2012). Direct evidence for inhibition of mitochondrial permeability transition pore opening by sevoflurane preconditioning in cardiomyocytes: comparison with cyclosporine A. Eur. J. Pharmacol. 675: 40-46.

Orriach JL, Aliaga MR, Ortega MG, Navarro MR, et al. (2013). Sevoflurane in intraoperative and postoperative cardiac surgery patients. Our experience in intensive care unit with sevoflurane sedation. Curr. Pharm. Des. 19: 3996-4002.

Peters JL, Sutton AJ, Jones DR, Abrams KR, et al. (2006). Comparison of two methods to detect publication bias in metaanalysis. JAMA 295: 676-680

Schmid E, Krajewski S, Bachmann D, Kurz J, et al. (2012). The volatile anesthetic sevoflurane inhibits activation of neutrophil granulocytes during simulated extracorporeal circulation. Int. Immunopharmacol. 14: 202-208.

Seki H, Fukunaga K, Arita M, Arai H, et al. (2010). The anti-inflammatory and proresolving mediator resolvin E1 protects mice from bacterial pneumonia and acute lung injury. J. Immunol. 184: 836-843.

Stang A (2010). Critical evaluation of the Newcastle-Ottawa scale for the assessment of the quality of nonrandomized studies in meta-analyses. Eur. J. Epidemiol. 25: 603-605.

Sunaga N, Kaira K, Tomizawa Y, Shimizu K, et al. (2014). Clinicopathological and prognostic significance of interleukin-8 expression and its relationship to KRAS mutation in lung adenocarcinoma. Br. J. Cancer 110: 2047-2053.

Wang YW, Yu WL, Xue YL, Weng YQ, et al. (2013). Effects of sevoflurane wash-in during cardiopulmonary bypass on inflammatory responses in patients undergoing coronary artery bypass grafting. Chin. J. Anesthesiol. 33: 1185-1187.

Watanabe K, Iwahara C, Nakayama H, Iwabuchi K, et al. (2013). Sevoflurane suppresses tumour necrosis factor-alphainduced inflammatory responses in small airway epithelial cells after anoxia/reoxygenation. Br. J. Anaesth. 110: 637-645.

Winterhalter M, Brandl K, Rahe-Meyer N, Osthaus A, et al. (2008). Endocrine stress response and inflammatory activation during CABG surgery. A randomized trial comparing remifentanil infusion to intermittent fentanyl. Eur. J. Anaesthesiol. 25: 326-335.

Xu B, Gao X, Xu J, Lei S, et al. (2011). Ischemic postconditioning attenuates lung reperfusion injury and reduces systemic proinflammatory cytokine release via heme oxygenase 1. J. Surg. Res. 166: e157-164.

Yin J, Wang SL and Liu XB (2014). The effects of general anaesthesia on memory in children: a comparison between propofol and sevoflurane. Anaesthesia 69: 118-123.

Zarogoulidis P, Katsikogianni F, Tsiouda T, Sakkas A, et al. (2014). Interleukin-8 and interleukin-17 for cancer. Cancer Invest. 32: 197-205.

Zhang J, Niu J and Yang J (2014). Interleukin-6, interleukin-8 and interleukin-10 in estimating the severity of acute pancreatitis: an updated meta-analysis. Hepatogastroenterology 61: 215-220.

Zhang XL, Wang Q, Zhou HY, Peng QM, et al. (2007). Effect of sevoflurane preconditioning on inflammatory response in patients undergoing cardiac surgery with cardiopulmonary bypass. Chin. J. Anesth. 27: 427-430.

Zhao J, Wang F, Zhang Y, Jiao L, et al. (2013). Sevoflurane preconditioning attenuates myocardial ischemia/reperfusion injury via caveolin-3-dependent cyclooxygenase-2 inhibition. Circulation 128: S121-S129.

Zhou SP, Liao WT, Yang LK and Sun L (2013). Effects of sevoflurane pretreatment on renal Src and FAK expression in diabetic rats after renal ischemia/reperfusion injury. Mol. Cell. Biochem. 384: 203-211.

Zintzaras E and loannidis JP (2005). HEGESMA: genome search meta-analysis and heterogeneity testing. Bioinformatics 21: 3672-3673. 